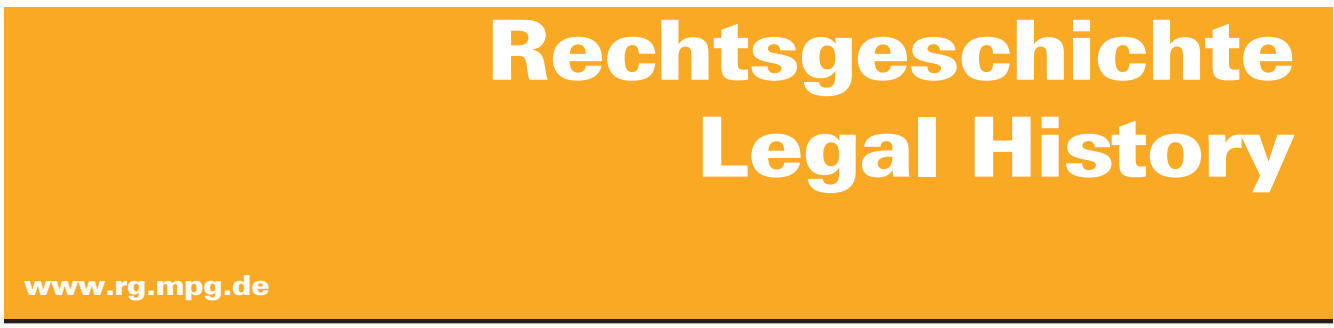

http://www.rg-rechtsgeschichte.de/rg23

Rg $22015 \quad 284-286$

Zitiervorschlag: Rechtsgeschichte - Legal History Rg 23 (2015)

http://dx.doi.org/10.12946/rg23/284-286

\title{
Kathrin Brunozzi
}

\section{Dreitausend Jahre Rechtsgeschichte}




\section{Kathrin Brunozzi Dreitausend Jahre Rechtsgeschichte*}

Die Einführung Heusers widmet sich etwa 3000 Jahren chinesischer Rechtsgeschichte vom 10. Jh. v. Chr. bis zur Gegenwart. Sie geht auf Einzelstudien zurück, die der Autor über etwa zehn Jahre hinweg veröffentlich hat. Das Buch besteht aus vier Kapiteln. Im ersten Kapitel befasst Heuser sich mit frühzeitlichen Rechtsordnungen (10.-1. Jh. v. Chr.), dehnt seine Betrachtungen aber meist bis ins 3. Jh. n. Chr. aus. Es geht vor allem um Inhalt und Gestalt des Rechtssystems sowie eine Einführung in die wichtigen Begriffe 法 (fa) und 禮 (li). Das zweite Kapitel ist im Wesentlichen Gesetzgebungsgeschichte. Ausgangspunkt der Betrachtung ist die Qin-Dynastie, die als erste Dynastie des chinesischen Kaiserreiches die Grundzüge des chinesischen Staatswesens schuf und sich am Legalismus orientierte. Die Herrscher der darauf folgenden Han-Dynastie legten ihrer Regierung konfuzianische Werte zugrunde. Es begann eine von Heuser als »Konfuzianisierung der Gesetze» (79) beschriebene Entwicklung, die in der TangDynastie "vollendet" wurde (100). Song-, Yuan-, Ming- und Qing-Dynastie hatten jeweils eigene Gesetzgebungswerke, die von unterschiedlichen Rechtsquellen ergänzt wurden. Das dritte Kapitel widmet Heuser dem 20. Jahrhundert, wobei er unter der Überschrift »Rechtsreform « in weiten Teilen die Gesetzgebungsgeschichte weitererzählt. Qing-Dynastie, Republik und Sozialismus bilden die großen Unterkapitel. Das Buch schließt mit einem Überblick über »Hundert Jahre Verfassungsreform« mit kurzen Abschnitten zur späten QingDynastie und frühen Republik, zur GuomingdangZeit und zu den letzten sechzig Jahren.

Heuser stellt die Entstehung, die Weiterentwicklung und die Modernisierung des chinesischen Rechts, vor allem der Gesetze, wohlstrukturiert und zugänglich dar. Es gelingt ihm, in die Gesetzgebungsgeschichte so einzuführen, dass auch der Leser ohne Vorkenntnisse des chinesischen Rechts gut folgen kann. Dabei deckt das
Buch einen Zeitraum von 3000 Jahren ab und thematisiert so viele Facetten und Aspekte der chinesischen Rechtsgeschichte, dass eine Besprechung nicht auf alle Besonderheiten eingehen kann. Die folgende Besprechung beschränkt sich daher auf vier Anmerkungen zu Heusers Grundriss.

Heuser beginnt seinen Grundriss mit einem systematischen Eingangskapitel. Im Mittelpunkt stehen dabei »Entstehung und Wandel eines eigenständigen Rechtsbegriffs« (19), der sich ihm zufolge im chinesischen Kontext herausgebildet hat und unter den zwei zentrale Normenkomplexe fallen: 法 (fa) und 禮 (li). Diese lassen sich aufgrund ihres »Verbindlichkeits- und Durchsetzungsanspruchs" von anderen Normen abgrenzen und »benennen« so »die Rechtsordnung« (19). An dieser Stelle drängen sich allerdings zwei Fragen an den Autor auf: Wieso soll der bloße Verbindlichkeits- und Durchsetzungsanspruch das rechtsdefinierende Merkmal bilden, das Rechtsnormen von anderen Normen unterscheidet? Und kann der so eingeführte, nicht näher qualifizierte »Verbindlichkeits- und Durchsetzungsanspruch « überhaupt als gemeinsamer Nenner für 法 (fa) und 禮 (li) fungieren? Hinsichtlich der ersten Fragen ist anzumerken, dass etwa auch Gepflogenheiten der Höflichkeit mit einem ebensolchen Anspruch auftreten könnten, ohne dass wir sie unter die Kategorie des Rechts subsumieren würden. Das von Heuser vorgeschlagene rechtsdefinierende Kriterium vermag demnach nicht zu erklären, wieso 法 (fa) als »Gesetzesnorm « und 禮 (li) als »Rechtsnorm « bezeichnet werden. Zudem macht er nicht klar, woher er dieses Kriterium gewinnt. Die Antwort auf die zweite Frage findet sich zum Teil in Heusers Ausführungen selbst. Diese deuten an, dass trotz des gemeinsamen »Verbindlichkeits- und Durchsetzungsanspruchs" gleichwohl gravierende Unterschiede zwischen 法 (fa) und 禮 (li) bestehen. So etwa verweisen die 法 (fa) vorrangig auf Strafen

\footnotetext{
Robert Heuser, Grundriss der Geschichte und Modernisierung des chinesischen Rechts (Studien zu Recht und Rechtskultur Chinas 2), Baden-Baden: Nomos 2013, 286 S., ISBN 978-3-8487-0781-2
} 
und gehören dem Bereich des Menschengemachten an (21). Die 禮 (li) hingegen werden auch als sakrale Regeln charakterisiert (23) und mit den leider nicht weiter spezifizierten Begriffen »Traditionsnormen« oder "Gewohnheitsrecht« (24) versuchsweise auf den Punkt gebracht. Diese Unterschiede werden dem Leser allerdings eher andeutend als auf systematische Klärung bedacht unterbreitet. Dabei gestatten chinesische theoretische Überlegungen zu den beiden Normenkomplexen, wie wir sie etwa in Texten wie dem Lunyu, dem Mengzi oder dem Xunzi finden, eine wesentlich differenzierte Abgrenzung der beiden Normenordnungen etwa hinsichtlich ihrer Entstehung, ihren Existenzbedingungen, ihrer normativen Quellen oder ihren Durchsetzungsmodalitäten. Heusers rechtsdefinierendes Kriterium vermag damit 法 (fa) und 禮 (li) nur oberflächlich und prima facie auf einen Nenner zu bringen. Bei näherem Hinsehen wird deutlich, dass mit beiden Normkomplexen sehr unterschiedliche »Verbindlichkeitsund Durchsetzungsansprüche« verbunden sind. Was zunächst als unter dem gleichen Kriterium vereint scheint, steht sich wohl fremder als vermutet gegenüber.

Im Zusammenhang mit 法 (fa) und 禮 (li) vertritt Heuser zudem die These, dass das Gesetzessystem in China schon sehr früh konfuzianisiert und ethisiert wurde. Dabei scheinen »das sich >konfuzianisierende oder sethisierende` Gesetzessystem " und die ")Vergesetzlichung des Konfuzianismus« zwei Seiten derselben Medaille zu sein. Ohne Heusers These im Grundtenor anzuzweifeln, lässt sie sich gleichwohl erweitern und präzisieren. Liang Zhiping etwa versucht in seinem 寻求自然秩序中的和谐 (Peking 1997, 326 ff.) zwischen den beiden Phänomenen der "Vergesetzlichung der Ethik « und der »Ethisierung des Gesetzes « stärker zu differenzieren. Mit der »Vergesetzlichung der Ethik « meint er wie Heuser die Imprägnierung der Gesetze durch die herrschende Moral. Mit der »Ethisierung der Gesetze« bezieht Liang sich allerdings differenzierter auf die Tatsache, dass bei der Anwendung der Gesetze weniger auf den Gesetzestext geachtet wurde, als vielmehr der Wille zur Umsetzung der Moral leitend war. Liang macht damit darauf aufmerksam, dass sich der Bezug zwischen Moral und Recht auf der Rechtsetzungs- und auf der Rechtsprechungsebene unterschiedlich darstellt: In der Rechtsetzung werden moralische Vorstellungen in rechtlichen Normen materialisiert. In der »Rechtsprechung « hin- gegen treten die Gesetze zuweilen in den Hintergrund und werden Urteile vorrangig auf moralische Prämissen gegründet. Liangs Ausführungen machen damit deutlich, dass eine Geschichte des chinesischen Rechts nicht ohne eine Betrachtung der Rechtspraxis auskommen kann. Sie ist einzubeziehen, wenn es um die Frage geht, wie Recht in China verstanden wurde und wie sich das Konzept historisch entwickelt hat. Bei Heuser, dessen "Grundriss der Geschichte [...] des Rechts« weitgehend Gesetzgebungsgeschichte darstellt, geschieht dies dagegen nur vereinzelt und nur insofern, als Auswirkungen von Rechtsprechung oder (fehlender) richterlicher Unabhängigkeit thematisiert werden (etwa 157 ff., 209).

Dass eine Geschichte des chinesischen Rechts mehr als nur stichprobenartig über die Gesetzgebungsgeschichte hinausgehen sollte, wird auch an Heusers Darstellung der letzten zwanzig Jahre hundertjähriger Rechtsreformen deutlich. So entgeht dem Leser von Heusers Buch, um nur dieses Beispiel zu nennen, die Debatte um eine Regierung basierend auf »rule of man«, »rule by law» und »rule of law«, die vor allem seit den Achtzigerjahren die Modernisierung des chinesischen Rechtssystem begleitet. Berücksichtigt man die Debatte als ganze, so wird deutlich, dass die Entwicklung hin zu einer Form der »rule of law» sich nicht in bloßer Weiterentwicklung "marxistischer Rechtstheorie « erschöpft (207, insbesondere Fn. 264). Vielmehr waren es vielfältige Prozesse und Akteure, u.a. Bemühungen von Rechtswissenschaftlern, die dazu führten, dass gegen Ende der Neunzigerjahre das Konzept einer »rule by law« schließlich - jedenfalls auf der Ebene des Verfassungstextes - durch eine "rule of law» ersetzt wurde (China's Journey toward the Rule of Law. Legal Reform 1978-2008, hg. v. Cai Dingjian/ Wang Chengguan, Leiden 2010). Dies wird als ein Meilenstein der Reform gewürdigt und ist sowohl die Folge als auch der Ausgangspunkt einer vielschichtigen, kontinuierlichen Auseinandersetzung damit, was eine Rechtsordnung ist, was von ihr zu erwarten ist und welchen Herausforderungen sie sich gegenüber sieht. Daran wird deutlich, dass die jüngste Rechtsgeschichte in Ländern wie China eben immer auch von breiteren intellektuellen Diskursen beeinflusst wird. Heusers Grundriss hätte diese Akteure einbeziehen können.

Abschließend bleibt zu betonen, dass Heuser seinen Lesern bei aller möglichen Kritik ohne Zweifel einen ersten Überblick über Rechtsquellen 
und Rechtsgeschichte verschafft. Dabei ist es vollkommen klar, dass eine dreitausendjährige Entwicklung auf 228 Seiten, die sich zudem nicht nur an Fachstudierende richten, an vielen Stellen nur oberflächlich dargestellt werden kann. Umso mehr kommt es darauf an, dem Leser nicht bloß aneinander gereihte historische Fakten zu präsentieren, sondern seinen Blick für Erkenntnisquellen und Entstehungsprozesse, aber auch für Forschungsdefizite und offene Fragen zu schärfen. Dies gelingt Heuser an vielen Stellen sehr gut. Die Ausführungen zum Qin-Kodex (72) geben z. B. einen lebendigen Überblick über Erkenntnismöglichkeiten und den Wandel, der sich in dem Blick auf eine Epoche ergeben kann, wenn neues Material gefunden wird. Die Darstellung zur chinesischen Fachbegriffsbildung, als es darum ging, ein Zivilgesetzbuch zu schaffen, führt dem Leser vor Augen, wie vieler "Zwischenschritte" eine Modernisierung des chinesischen Rechts bedurfte. Heuser haucht dem abstrakt anmutenden Begriff der Rechtsreform dadurch in willkommenem Maße Leben ein. Es gelingt ihm zudem immer wieder, aus der Menge der Informationen sinnvolle Kategorien zu bilden, Kontinuitäten aufzuzeigen und Linien zu beschreiben. Gleichzeitig ist anzumerken: Ergänzende eigene Recherche zur Geschichte des chinesischen Rechts erspart Heusers Grundriss nicht. Den Einstieg in eine solche Recherche erleichtert Heuser in erster Linie hinsichtlich der Gesetzestexte und der darauf bezogenen zeitgenössischen Quellen, insbesondere des beginnenden 20. Jahrhunderts. An anderer Stelle müsste er dem Leser - dem Zweck einer Einführung entsprechend - mehr an die Hand geben. Eine stärkere Einbeziehung amerikanischer und asiatischer Forschungsergebnisse des 21. Jahrhunderts würde den Zugang zu Problemkreisen ermöglichen, die durch Heusers Quellenauswahl in seinem Grundriss untergehen. Das Fazit muss also lauten, dass Heusers Werk einen guten Überblick gibt. Es lohnt sich aber auf eigene Faust über seine Ausführungen hinauszuschauen.

\section{Michael Stolleis}

\section{Juristen und »Juristen«"}

Die Lektüre des Buchs beginnt mit dem Titel. Er ist offenbar bewusst vage gehalten. »Die Juristen «, als Gattung oder Stand, als Denktypus, als wissenschaftliche Formation? Geht es um Juristen aller Herren und Länder? Und was bedeutet »kritische Geschichte « - ist eine unkritische denkbar? Handelt es sich um eine soziologische historische Studie zur Ausbreitung der Juristen und eine Analyse ihres Einflusses? »The Jurists « ist also ein anspruchsvolles Portal, hinter dem sich jedenfalls kein Juristenlexikon verbirgt. Treten wir also ein und sehen wir zunächst, welchen Ballast Gordley abwerfen musste, um sein Schiff flott zu bekommen.
Nicht behandelt werden die »Juristen « der ganzen Welt, unter welchem Namen sie auch immer als Spezialisten für die Lösung von zwischenmenschlichen Konflikten auftreten mögen. "Jurists« sind (im Gegensatz zu den »lawyers«, »barristers" oder »solicitors«) die universitären Vertreter der iurisprudentia. Nicht gehandelt wird also von richtenden Dorfältesten oder Ratsherren, von juristisch ausgebildeten Syndici, Anwälten und Notaren, Hof- und Staatsräten und anderen. Es geht auch nicht um eine vergleichende Sozialgeschichte des akademischen Juristenpersonals, die zeigen könnte, wie sich Verhaltenskodices der Rechtslehrer ausbilden, wie sie eine Vorrangstellung vor den

\footnotetext{
* James Gordeny, The Jurists. A Critical History, Oxford: Oxford University Press 2013, XI, 320 S.,

ISBN 978-0-19-968939-2
} 